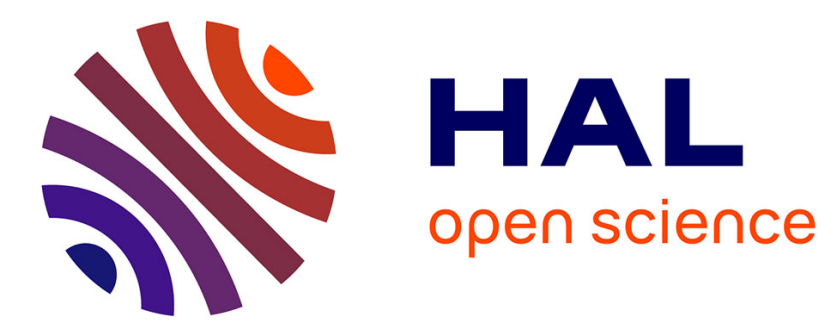

\title{
La démographie de la France en 2011: continuité et inflexion
}

\author{
Gérard-François Dumont
}

\section{To cite this version:}

Gérard-François Dumont. La démographie de la France en 2011: continuité et inflexion. Population et avenir, 2012, 707, pp.18-19. 10.3917/popav.707.0018 . halshs-00770090

\section{HAL Id: halshs-00770090 \\ https://shs.hal.science/halshs-00770090}

Submitted on 16 Oct 2019

HAL is a multi-disciplinary open access archive for the deposit and dissemination of scientific research documents, whether they are published or not. The documents may come from teaching and research institutions in France or abroad, or from public or private research centers.
L'archive ouverte pluridisciplinaire HAL, est destinée au dépôt et à la diffusion de documents scientifiques de niveau recherche, publiés ou non, émanant des établissements d'enseignement et de recherche français ou étrangers, des laboratoires publics ou privés. 


\section{La démographie de la France en 2011 : continuité et inflexion}

A priori, les résultats démographiques de la France de l'année 2011 sont dans la ligne des années précédentes. Toutefois, des inflexions se dessinent. Et les différences de fécondité au sein des populations de la France sont mises en évidence.

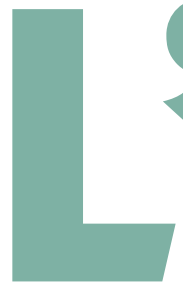

par GérardFrançois DUMONT
1. Paris, Frank, «Et Mayotte... nouveau Dom depuis mars 2011 Population \& Avenir, $n^{\circ} 704$, septembreoctobre 2011.

2. Dumont, Gérard-François, « La démographi de la France en 2010 le double paradoxe" Population \& Avenir, $n^{\circ} 702$, mars-avril 2011

3. Selon une étude Gallup conduite en 2011 dans 53 pays. Un sondage Ifop de janvier 2012 montre que $65 \%$ des Français craignent pour leur avenir et celui de leurs enfants.

\section{Un paradoxe confiirmé}

2011 se caractérise, pour la population de la France, par le passage au-dessus du chiffre de 65 millions, additionnant le nombre d'habitants de la France métropolitaine et celui des 4 départements d'outre-mer. Toutefois, l'intitulé « France » utilisé par l'Insee ne prend pas en compte le nouveau et cinquième département d'outre-mer de l'année 2011, Mayotte ${ }^{1}$.

Les ordres de grandeur de la natalité, de la mortalité et du solde migratoire de l'année 2011 apparaissent semblables à ceux des deux années précédentes. Cela n'est pas illogique dans la mesure où les processus démographiques s'inscrivent souvent dans la durée et où les effets de la crise économique se révèlent, comme en 2010, quasiment neutres sur les évolutions démographiques. Le paradoxe français ${ }^{2}$ se confirme avec le peuple champion du monde du pessimisme économique, plus pessimiste que les Irakiens, les Afghans ou les Pakistanais ${ }^{3}$, et pourtant, la fécondité la plus élevée d'Europe (2,01 enfants par femme en 2011) avec l'Irlande et l'Islande.

\section{Des inflexions notables}

Toutefois, l'année 2011 marque d'incontestables inflexions. La légère baisse des naissances marie la faible diminution de la fécondité avec celle du nombre de femmes en âge de procréer. La hausse des décès est la conséquence directe du vieillissement de la population, même si cette hausse est minorée par l'augmentation de l'espérance de vie. Et cette dernière profite davantage au sexe masculin qu'au sexe féminin. Toutefois, l'espérance de vie des femmes à la naissance demeure supérieure de 6,6 années à celles des hommes.

Enfin, les différences de fécondité selon les populations de la France demeurent significatives. La fécondité des mères de nationalité étrangère hors de l'Union européenne est le double de la moyenne nationale.

\section{FIg.1. L'INdice de fÉCONdITÉ En France} SELON LE PAYS DE NAISSANCE DE LA MÈRE (NOMBRE D'ENFANTS PAR FEMME)

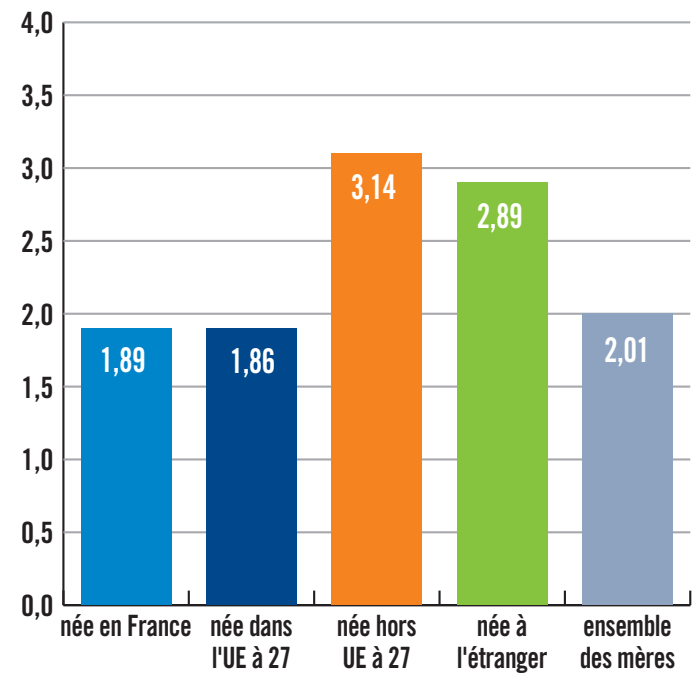

FIG. 2. L'INDICE DE FÉCONDITÉ EN

FRANCE SELON LA NATIONALITÉ DE LA MÈRE (NOMBRE D'ENFANTS PAR FEMME)

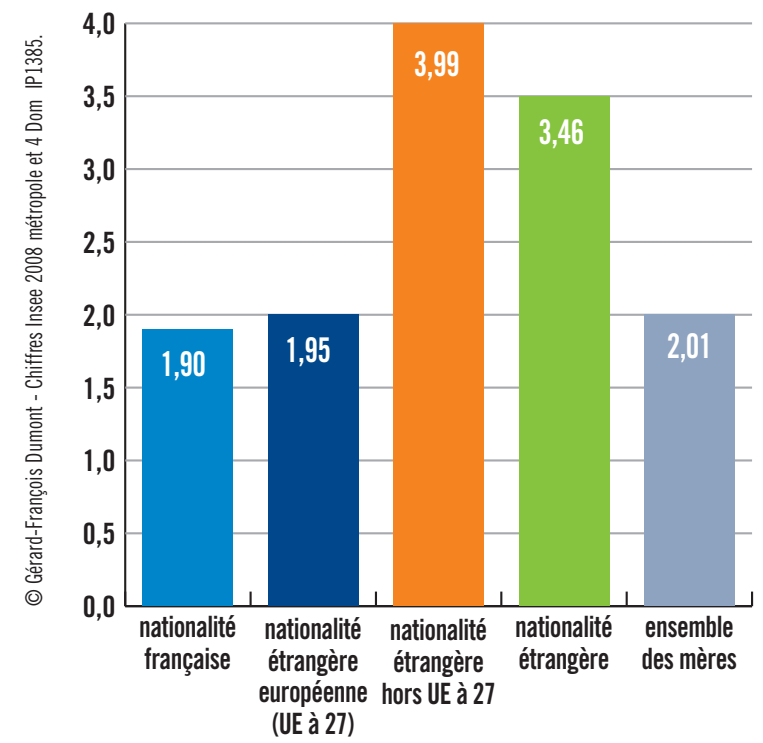


LE MOUVEMENT DE LA POPULATION DE LA FRANCE EN OUELOUES CHIFERES

\section{A. France métropolitaine}

\begin{tabular}{|c|c|c|c|c|c|c|c|c|}
\hline \multirow[b]{2}{*}{ Année } & \multirow{2}{*}{$\begin{array}{c}\text { Population } \\
\text { moyenne }\end{array}$} & \multicolumn{4}{|c|}{ Chiffres en milliers } & \multicolumn{3}{|c|}{ Taux pour 1000 habitants } \\
\hline & & $\begin{array}{c}\text { Naissances } \\
\text { vivantes }\end{array}$ & Décès & $\begin{array}{c}\text { Solde } \\
\text { naturel }\end{array}$ & $\begin{array}{c}\text { Solde } \\
\text { migratoire }\end{array}$ & Natalité & Mortalité & $\begin{array}{c}\text { Accroisse- } \\
\text { ment naturel }\end{array}$ \\
\hline 1980 & 53880 & 800,4 & 547,1 & $+253,3$ & +44 & 14,9 & 10,2 & $+4,7$ \\
\hline 1985 & 55284 & 768,4 & 552,5 & $+215,9$ & +38 & 13,9 & 10,0 & $+3,9$ \\
\hline 1990 & 56708 & 762,4 & 526,2 & $+236,2$ & +80 & 13,4 & 9,3 & $+4,1$ \\
\hline 1995 & 57844 & 729,6 & 531,6 & $+198,0$ & +40 & 12,6 & 9,2 & $+3,4$ \\
\hline 1999 & 58677 & 744,8 & 537,7 & $+207,1$ & +60 & 12,7 & 9,2 & $+3,5$ \\
\hline 2000 & 59062 & 774,8 & 530,9 & $+243,9$ & +70 & 13,1 & 9,0 & $+4,1$ \\
\hline 2001 & 59476 & 770,9 & 531,1 & $+239,8$ & +85 & 13,0 & 8,9 & $+4,1$ \\
\hline 2002 & 59894 & 761,6 & 535,1 & $+226,5$ & +95 & 12,7 & 8,9 & $+3,8$ \\
\hline 2003 & 60304 & 761,5 & 552,3 & $+209,2$ & +100 & 12,6 & 9,2 & $+3,4$ \\
\hline 2004 & 60734 & 767,8 & 509,4 & $+258,4$ & +105 & 12,7 & 8,4 & $+4,3$ \\
\hline 2005 & 61181 & 774,4 & 527,5 & $+246,9$ & +95 & 12,7 & 8,6 & $+4,1$ \\
\hline 2006 & 61597 & 796,9 & 516,4 & $+280,5$ & +115 & 12,9 & 8,4 & $+4,5$ \\
\hline 2007 & 61963 & 786,0 & 521,0 & $+265,0$ & +75 & 12,7 & 8,4 & $+4,3$ \\
\hline 2008 & 62300 & 796,0 & 532,1 & $+263,9$ & +67 & 12,9 & 8,6 & $+4,3$ \\
\hline 2009 & 62636 & 793,4 & 538,1 & $+255,3$ & +70 & 12,7 & 8,6 & $+4,1$ \\
\hline 2010 (p) & 62967 & 802,0 & 540,5 & $+261,7$ & +75 & 12,7 & 8,6 & $+4,2$ \\
\hline $2011(p)$ & 63294 & 797,0 & 544,0 & $+253,0$ & +80 & 12,6 & 8,6 & $+4,0$ \\
\hline
\end{tabular}

(p) Résultats provisoires

B. France métropolitaine + Dom (Guadeloupe*, Guyane, Martinique, Réunion)

\begin{tabular}{|c|c|c|c|c|c|c|c|c|}
\hline \multirow[b]{2}{*}{ Année } & \multirow{2}{*}{$\begin{array}{l}\text { Population } \\
\text { moyenne }\end{array}$} & \multicolumn{4}{|c|}{ Chiffres en milliers } & \multicolumn{3}{|c|}{ Taux pour 1000 habitants } \\
\hline & & $\begin{array}{l}\text { Naissances } \\
\text { vivantes }\end{array}$ & Décès & $\begin{array}{c}\text { Solde } \\
\text { naturel }\end{array}$ & $\begin{array}{c}\text { Solde } \\
\text { migratoire }\end{array}$ & Natalité & Mortalité & $\begin{array}{l}\text { Accroisse- } \\
\text { ment naturel }\end{array}$ \\
\hline 1999 & 60316 & 775,8 & 547,3 & $+228,5$ & +61 & 12,9 & 9,1 & $+3,8$ \\
\hline 2000 & 60725 & 807,4 & 540,6 & $+266,8$ & +71 & 13,3 & 8,9 & $+4,4$ \\
\hline 2001 & 61163 & 803,2 & 541,0 & $+262,2$ & +87 & 13,1 & 8,8 & $+4,3$ \\
\hline 2002 & 61605 & 792,7 & 545,2 & $+247,5$ & +97 & 12,9 & 8,9 & $+4,0$ \\
\hline 2003 & 62038 & 793,0 & 562,5 & $+230,5$ & +102 & 12,8 & 9,1 & $+3,7$ \\
\hline 2004 & 62491 & 799,4 & 519,5 & $+279,9$ & +105 & 12,8 & 8,3 & $+4,5$ \\
\hline 2005 & 62959 & 806,8 & 538,1 & $+268,7$ & +92 & 12,9 & 8,6 & $+4,3$ \\
\hline 2006 & 63394 & 829,3 & 526,9 & $+302,4$ & +112 & 13,1 & 8,3 & $+4,8$ \\
\hline 2007 & 63782 & 818,7 & 531,2 & $+287,5$ & +74 & 12,8 & 8,3 & $+4,5$ \\
\hline 2008 & 64134 & 828,4 & 542,6 & $+285,8$ & +57 & 12,9 & 8,5 & $+4,4$ \\
\hline 2009 & 64477 & 824,6 & 548,5 & $+276,1$ & +67 & 12,8 & 8,5 & $+4,3$ \\
\hline $2010(p)$ & 64838 & 832,8 & 551,2 & $+281,6$ & +62 & 12,8 & 8,4 & $+4,4$ \\
\hline 2011 (p) & 65175 & 827,0 & 555,0 & $+272,0$ & +77 & 12,7 & 8,5 & $+4,2$ \\
\hline \multicolumn{9}{|c|}{ 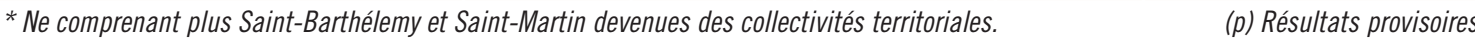 } \\
\hline \multicolumn{9}{|c|}{ Solution de la page 2} \\
\hline \multicolumn{9}{|c|}{$\begin{array}{l}\text { Le suicide des personnes âgées. Le taux de suicide est nettement plus élevé chez les personnes âgées de } 85 \text { à } 94 \text { ans que la moyenne de la population } \\
\text { de la France, contrairement àce qu'on pense généralement. Mais le pourcentage du suicide comme cause de déces est plus faible chez les } 85 \text { à } 94 \text { ans } \\
\text { (3,5 suicides pour mille décès) que dans la moyenne nationale (19,4 suicides pour mille décès). Selon les derniers chiffres disponibles, la France compte } \\
\text { chaque année un peu plus de } 500 \text { suicides chez les } 85 \text { à } 94 \text { ans et plus de } 10000 \text { pour l'ensemble de la population. }\end{array}$} \\
\hline
\end{tabular}

